\title{
Topological fluid dynamics of interfacial flows
}

\section{Brøns, Morten}

Published in:

Physics of Fluids

Link to article, DOI:

$10.1063 / 1.868163$

Publication date:

1994

\section{Document Version}

Publisher's PDF, also known as Version of record

Link back to DTU Orbit

\section{Citation (APA):}

Brøns, M. (1994). Topological fluid dynamics of interfacial flows. Physics of Fluids, 6(8), 2730-2737. https://doi.org/10.1063/1.868163

\section{General rights}

Copyright and moral rights for the publications made accessible in the public portal are retained by the authors and/or other copyright owners and it is a condition of accessing publications that users recognise and abide by the legal requirements associated with these rights.

- Users may download and print one copy of any publication from the public portal for the purpose of private study or research.

- You may not further distribute the material or use it for any profit-making activity or commercial gain

- You may freely distribute the URL identifying the publication in the public portal

If you believe that this document breaches copyright please contact us providing details, and we will remove access to the work immediately and investigate your claim. 


\title{
Topological fluid dynamics of interfacial flows
}

\author{
Morten Brøns \\ Mathematical Institute, The Technical University of Denmark, Building 303, DK-2800 Lyngby, Denmark
}

(Received 15 December 1993; accepted 5 April 1994)

\begin{abstract}
The topological description of flows in the vicinity of a solid boundary, that is familiar from the aerodynamics literature, has recently been extended to the case of flow at a liquid-gas interface or a free surface by Lugt [Phys. Fluids 30, 3647 (1987)]. Lugt's work is revisited in a more general setting, including nonconstant curvature of the interface and gradients of surface tension, using tools of modern nonlinear dynamics. Bifurcations of the flow pattern occur at degenerate configurations. Using the theory of unfolding, this paper gives a complete description of the bifurcations that depend on terms up to the second order. The general theory of this paper is applied to the topology of streamlines during the breaking of a wave and to the flow below a stagnant surface film.
\end{abstract}

\section{INTRODUCTION}

Topological fluid dynamics is the study of qualitative properties of fluid flows. In Ref. 1 a number of recent applications of topology to the physics of fluids can be found. In the present paper, we consider a classical aspect of topological fluid dynamics, that is, the study of local, qualitative properties of steady flows based on Taylor expansions of the governing fields. Oswatitsch ${ }_{2}{ }^{2}$ Legendre, ${ }^{3}$ and Lighthill ${ }^{4}$ have used this point of view to describe the flow in the vicinity of a point of separation of a solid boundary.

Developments during the last decades in the qualitative theory of dynamical systems, in particular bifurcation theory, have shed new light on the subject; see, for example, the review by Tobak and Peake ${ }^{5}$ and the monograph by Bakker. ${ }^{6}$ Also, modern computer algebra systems are most useful in keeping track of the many series coefficients involved (cf. Perry and Chong ${ }^{7}$ ).

Lugt was the first to apply topological fluid mechanics to flows near fluid interfaces in two dimensions ${ }^{8}$ with some generalizations to three dimensions." In the present paper we reconsider the two-dimensional problem in a more general setting, first of all avoiding Lugt's assumption that the interface is circular, and allowing the interface to take an arbitrary shape.

Bifurcations in the flow patterns occur when parameters are varied across certain degenerate values. We give a complete description of the bifurcations-or, in the language of singularity theory, unfoldings - that depend on expansion coefficients up to second order. This complements similar studies by Dallmann ${ }^{10}$ and Bakker ${ }^{6}$ for the flow close to a planar wall.

The general theory is used to study the change of the streamline pattern as a two-dimensional wave steepens and breaks. Our analysis shows that the series of patterns of mean streamlines conjectured by Banner and Melville ${ }^{11}$ agrees with the simplest possible combination that obtains from an analysis of a steady flow.

A result of our investigation is that possible gradients of surface tension enter at an early order. Such gradients occur in the presence of a contaminant film on the surface. In the flow below a stagnant film, the Reynolds Ridge, ${ }^{12}$ we show that separation or attachment must occur at points on the interface where the surface tension has a maximum or a minimum.

\section{BASIC EQUATIONS AND BOUNDARY CONDITIONS}

We consider the steady, two-dimensional flow of two immiscible fluids. The interface is given by $x_{2}=f\left(x_{1}\right)$ with fluid 1 below and fluid 2 above. We assume that the coordinate system is translated and rotated such that $f(0)=f^{\prime}(0)=0$. See Fig. 1 . For fluid $k,(k=1,2)$, the continuity and Navier-Stokes equations in component notation are

$$
\begin{aligned}
& \frac{\partial u_{i}^{(k)}}{\partial x_{i}}=0, \\
& u_{j}^{(k)} \frac{\partial u_{i}^{(k)}}{\partial x_{j}}+\frac{1}{\rho_{k}} \frac{\partial p^{(k)}}{\partial x_{i}}=\frac{\mu_{k}}{\rho_{k}} \Delta u_{i}^{(k)}+g r_{i} .
\end{aligned}
$$

Here, $\left(u_{1}^{(k)}, u_{2}^{(k)}\right)$ are the velocity components, $p^{(k)}$ is the pressure, $\mu_{k}, \rho_{k}, g$ are the viscosity, density, and acceleration of gravity, and $\mathbf{r}=(\cos \alpha, \sin \alpha)$ is the vertical direction vector, $\alpha$ being the angle between the $x_{1}$-axis and the vertical direction.

The normal vector, the tangential vector, and the radius of curvature of the interface at $\left(x_{1}, f\left(x_{1}\right)\right)$ are

$$
\begin{aligned}
& n=\left(1+f^{\prime}\left(x_{1}\right)^{2}\right)^{-1 / 2}\left(-f^{\prime}\left(x_{1}\right), 1\right), \\
& t=\left(1+f^{\prime}\left(x_{1}\right)^{2}\right)^{-1 / 2}\left(1, f^{\prime}\left(x_{1}\right)\right), \\
& R=\frac{\left(1+f^{\prime}\left(x_{1}\right)^{2}\right)^{3 / 2}}{f^{\prime \prime}\left(x_{1}\right)}
\end{aligned}
$$

The kinematic boundary condition at the interface is that the velocity field must be parallel to the interface,

$$
n_{i} u_{i}^{(k)}=0 \text {. }
$$

At the interface, surface tension $\sigma$ contributes to the stress by

$$
\boldsymbol{\tau}_{\mathrm{s}}=\frac{\sigma}{R} n+\frac{\partial \sigma}{\partial s} t
$$

where $s$ measures arclength of the interface. Since

$$
d s^{2}=\left(1+f^{\prime}\left(x_{1}\right)^{2}\right) d x_{1}^{2},
$$




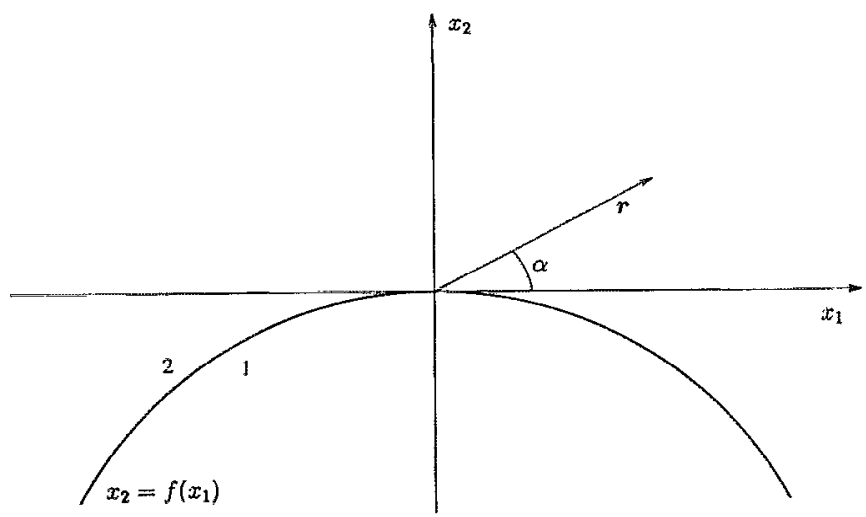

FIG. 1. The geometry of the problem. The interface is given by the curve $x_{2}=f\left(x_{1}\right)$ with fluid 1 below and fluid 2 above. The vector $\mathbf{r}$ denotes the vertical direction.

we get the dynamic boundary conditions at the interface

$$
\begin{aligned}
& t_{i} u_{i}^{(1)}=t_{i} u_{i}^{(2)}, \\
& n_{i} \tau_{i j}^{(1)} t_{j}+\frac{\partial \sigma}{\partial x_{1}}\left(1+f^{\prime}\left(x_{1}\right)^{2}\right)^{-1 / 2}=n_{i} \tau_{i j}^{(2)} t_{j}, \\
& -p^{(1)}+n_{i} \tau_{i j}^{(1)} n_{j}+\frac{\sigma}{R}=-p^{(2)}+n_{i} \tau_{i j}^{(2)} n_{j} .
\end{aligned}
$$

Equation (9) expresses continuity of the tangential component of velocity across the interface, while Eqs. (10)-(11) are continuity of tangential and normal stresses. The viscous strcss tensor is

$$
\tau_{i j}^{(k)}=\mu_{k}\left(\frac{\partial u_{i}^{(k)}}{\partial x_{j}}+\frac{\partial u_{j}^{(k)}}{\partial x_{i}}\right) .
$$

If the stresses in fluid 2 are neglected, the interface is a free surface. Then continuity of tangential velocity cannot be imposed, and continuity of stresses reduces to

$$
\begin{aligned}
& n_{i} \tau_{i j}^{(1)} t_{j}+\frac{\partial \sigma}{\partial x_{1}}\left(1+f^{\prime}\left(x_{1}\right)^{2}\right)^{-1 / 2}=0, \\
& -p^{(1)}+n_{i} \tau_{i j}^{(1)} n_{j}+\frac{\sigma}{R}=0 .
\end{aligned}
$$

\section{FLOW TOPOLOGY IN ONE FLUID}

\section{A. Taylor expansions}

To study the local properties of the flow, the fields in each fluid are expanded in Taylor series based at the origin,

$$
\begin{aligned}
& u_{1}^{(k)}=\sum_{n+m=0}^{\infty} a_{n m}^{(k)} x_{1}^{n} x_{2}^{m}, \quad u_{2}^{(k)}=\sum_{n+m=0}^{\infty} b_{n m}^{(k)} x_{1}^{n} x_{2}^{m}, \\
& p^{(k)}=\sum_{n+m=0}^{\infty} c_{n m}^{(k)} x_{1}^{n} x_{2}^{m} .
\end{aligned}
$$

In the present section we consider the flow in one fluid only and drop the superscripts for convenience. We provisionally think of the interface $x_{2}=f\left(x_{1}\right)$ as an internal streamline.
Having found possible flow topologies on each side of this streamline, we will later use the dynamic boundary conditions (9)-(11) or (13)-(14) to patch together these configurations across the interface.

Thus, we here consider a flow satisfying Eqs. (1)-(2) and the kinematic boundary condition (6) only. Inserting the expansions (15)-(16) here, a number of equations involving the expansion coefficients can be derived. There are, however, more unknowns than equations; the missing information is in boundary conditions away from the interface.

However, some of the coefficients can be determined. We first note that all $b_{n m}$ can be eliminated, since

$$
b_{n m}=-\frac{n+1}{m} a_{n+1 m+1}
$$

follows from continuity. Assuming that the interface is smooth, we can write

$$
f\left(x_{1}\right)=\sum_{n=2}^{\infty} s_{n} x_{1}^{n} .
$$

The remaining $b_{n 0}$ can be found from Eq. (6) expressed in terms of $a_{n m}$ and $s_{n}$.

Furthermore, all derivatives of the pressure can be eliminated using the Navier-Stokes equations. This leaves $p$ determined except for its (arbitrary) value at the origin, $c_{00}$. Finally, some of the $a_{n m}$ can be eliminated from the NavierStokes equations, the lowest order term being of third order. As mentioned in the Introduction, we will only consider truncations of (15) up to the second order. Thus, the NavierStokes equations do not give us any direct information on the coefficients $a_{n m}$, and the results in this section depend only on the continuity equation (1).

Note, that the coefficients $a_{n m}, b_{n m}$ are directly related to the viscous stress tensor (12) and its derivatives evaluated at the origin. For example,

$\tau_{12}=\tau_{21}=\mu\left(a_{01}+b_{10}\right), \tau_{11}=2 \mu a_{10}, \quad \tau_{22}=2 \mu b_{01}$.

Truncating the velocity field after second-order terms, the resulting equations for the streamlines are

$$
\begin{aligned}
\dot{x}_{1}=u_{1}= & a_{00}+a_{10} x_{1}+a_{01} x_{2}+a_{20} x_{1}^{2}+a_{11} x_{1} x_{2}+a_{02} x_{2}^{2}, \\
\dot{x}_{2}=u_{2}= & 2 a_{00} s_{2} x_{1}-a_{10} x_{2}+3\left(a_{10} s_{2}+a_{00} s_{3}\right) x_{1}^{2} \\
& -2 a_{20} x_{1} x_{2}-\frac{a_{11}}{2} x_{2}^{2} .
\end{aligned}
$$

\section{B. Regular and degenerate singular points}

If $a_{00}=0$, the origin is a singular point for (20)-(21). Linearizing, the system matrix is

$$
J=\left(\begin{array}{cc}
a_{10} & a_{01} \\
0 & -a_{10}
\end{array}\right) .
$$

Assuming first that $a_{10}<0$, we see that the origin is a saddle with eigenvalues $a_{10},-a_{10}$, the stable direction being determined by the eigenvector $(1,0)$ corresponding to the flow on the interface. The unstable direction is given by 


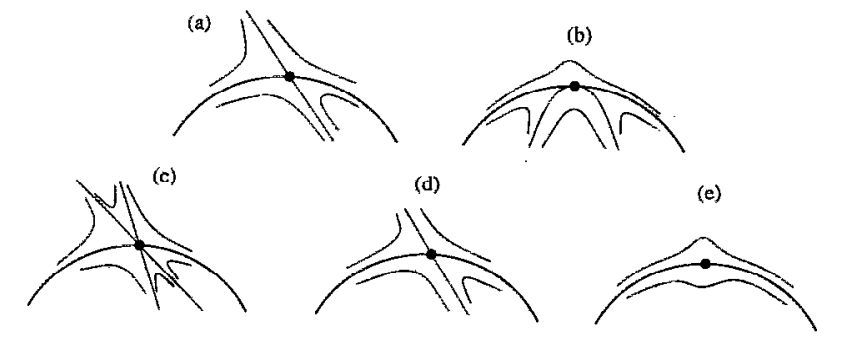

FIG. 2. Streamlines in the vicinity of a singular point on the distinguished streamline $x_{2}=f\left(x_{1}\right)$. All figures are shown in the physical $\left(x_{1}, x_{2}\right)$-plane. (a) Nondegenerate point, $a_{10} \neq 0$. (b) Simple degeneracy, $\lambda=0, \nu>0$ ). The rest of the figures show the double degeneracy $\lambda=\nu=0$. (c) $D>0$. (d) $D=0$. (e) $D<0$.

the other eigenvector, $\left(a_{01},-2 a_{10}\right)$. Thus, the origin is a stagnation point, and the slope of the dividing streamline is

$$
\tan \theta=-2 \frac{a_{10}}{a_{01}}=-\frac{\tau_{11}}{\tau_{12}}
$$

for $a_{01} \neq 0$. If $a_{01}=0, \theta=\pi / 2$. See Fig. 2(a). If $a_{10}>0$, the geometry is the same but with the flow reversed.

If $a_{10}=0$, zero is a double eigenvalue of $J$, and higher order terms determine the topology. In the following analysis, it will be convenient to introduce new variables

$$
\xi=x_{1}-x_{0}, \quad \eta=x_{2}-f\left(x_{1}\right) .
$$

The new variables $(\xi, \eta)$ have the interface as the line $\eta=0$, and the origin is translated $x_{0}$ along the $x_{1}$-axis. This transforms $(20)-(21)$ to

$$
\begin{aligned}
\dot{\xi}= & a_{00}+a_{10} x_{0}+d_{2} x_{0}^{2}+\left(a_{10}+2 d_{2} x_{0}\right) \xi \\
& +\left(a_{01}+a_{11} x_{0}\right) \eta+d_{2} \xi^{2}+a_{11} \xi \eta+a_{02} \eta^{2} \\
\dot{\eta}= & -\eta\left(a_{10}+2 d_{2} x_{0}+2 d_{2} \xi+\frac{a_{11}}{2} \eta\right)
\end{aligned}
$$

where

$$
d_{2}=a_{20}+s_{2} a_{01} \text {. }
$$

Now assume $d_{2} \neq 0$. This is a nondegeneracy condition which we will assume from now on, unless explicitly stated, whenever second-order terms are involved. Without loss of generality we will even assume $d_{2}>0$, since, if necessary, this may be obtained by reversal of the sense of time. By choosing

$$
x_{0}=-\frac{a_{10}}{2 d_{2}},
$$

the $\xi$-term in (24) and the $\eta$-term in (25) vanish. We then get the system

$$
\begin{aligned}
& \dot{\xi}=\lambda+\nu \eta+d_{2} \xi^{2}+a_{11} \xi \eta+a_{02} \eta^{2}, \\
& \dot{\eta}=-\eta\left(2 d_{2} \xi+\frac{a_{11}}{2} \eta\right),
\end{aligned}
$$

with the new parameters

$$
\lambda=a_{00}-\frac{1}{4} \frac{a_{10}^{2}}{d_{2}}, \quad \nu=a_{01}-\frac{a_{11} a_{10}}{2 d_{2}} .
$$

Thus, by an appropriate choice of $x_{0}$, we have effectively reduced the number of parameters by one, since $a_{00}$, $a_{10}$, and $a_{01}$ only appear in the combinations $\lambda, \nu$. Bakker ${ }^{6}$ refers to this as the movement principle.

In the degenerate case $a_{00}=a_{10}=0$ we get $\lambda=0, \nu=a_{01}$. Then the origin of $(27)-(28)$ is a singular point with linearization

$$
J=\left(\begin{array}{ll}
0 & \nu \\
0 & 0
\end{array}\right)
$$

If $\nu \neq 0$, zcro is an eigenvalue with algebraic multiplicity two and geometric multiplicity one. This is a simple degeneracy since only one parameter takes a degenerate value. Andronov's classification scheme ${ }^{13,6}$ gives an algorithm to determine the local phase portrait. Using this, a simple calculation shows that the origin is a topological saddle. Thus, a number of separatrices (dividing streamlines) meet at the origin. These can be found from the Ansatz

$$
\eta=A \xi^{n}+\Theta\left(\xi^{n+1}\right)
$$

as trajectories for (27)-(28). For each $n, A=0$ is a solution, representing the interface. The lowest $n$ for which trajectories (31) exist determines the number and local shape of the separatrices.

In the present case, $n=2$, and

$$
A=-\frac{d_{2}}{\nu} \text {. }
$$

This represents a locally parabolic separatrix which is below the interface if $d_{2}$ and $\nu$ have the same sign and above the interface if $d_{2}$ and $\nu$ have opposite signs. See Fig. 2(b).

A double degeneracy occurs when $\lambda=\nu=0$. Here the linear part $J$ of (27)-(28) vanishes. Such systems are traditionally studied by blow-up techniques, i.e., singular transformations of coordinates, ${ }^{13,6}$ but we may also proceed as above to find possible separatrices. Indeed, trajectories of the form (31) with $n=1$ exist when

$$
2 a_{02} A^{2}+3 a_{11} A+6 d_{2}=0 .
$$

Imposing here the further nondegeneracy condition $a_{02}$ $\neq 0$, the discriminant of the second-order equation (32) is

$$
D=9 a_{11}^{2}-48 a_{02} d_{2} \text {. }
$$

If $D>0$, (32) has two solutions, each corresponding to locally linear separatrices. If $D=0$, one locally linear separatrix exist. If $D<0$, there are no separatrices except the interface. See Fig. 2(c)-2(e).

Note, that while cases (a) and (d) are topologically alike, they are geometrically different. In case (a), the leading order terms in the streamfunction are quadratic, and the streamlines are locally hyperbolas. In case (d), the leading order terms are cubic, and the streamlincs are third-order curves.

\section{Unfolding the simple degeneracy $\lambda=0$}

To study the dynamics when the parameters take values close to the degenerate cases, we first identify all singular points of the system (27)-(28). 

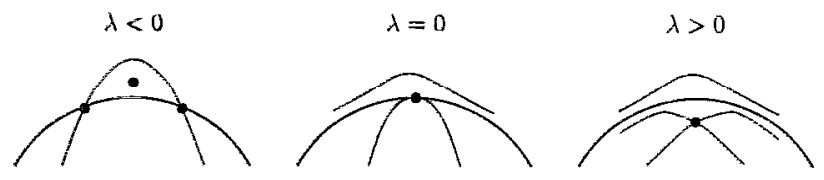

FIG. 3. Unfolding of the simple degeneracy $\lambda=0, \nu>0$.

On the interface where $\eta=0$, the singular points are given by

$$
\xi= \pm \sqrt{-\frac{\lambda}{d_{2}}}
$$

Thus, two singular points exist when $\lambda$ has opposite sign of $d_{2}$. As $\lambda$ approaches 0 they merge and disappear when $\lambda$ changes sign. A stability analysis show that these points are saddles when $\lambda \neq 0$, with eigenvalues $\pm \sqrt{-\lambda d_{2}}$. isfy

The singular points which may be off the interface sat-

$$
\begin{aligned}
& \xi=-\frac{1}{4} \frac{a_{11}}{d_{2}} \eta, \\
& -\frac{D}{48 d_{2}} \eta^{2}+\nu \eta+\lambda=0,
\end{aligned}
$$

where $D$ is given by (33).

Now consider $\lambda$ close to zero and $\nu \neq 0$. Then (35) has one solution which is close to the origin,

$$
\eta=-\frac{\lambda}{\nu}+\sigma\left(\lambda^{2}\right)
$$

Evaluating the Jacobian $J$ of (27)-(28) at the singular point one finds

$$
\operatorname{det} J=-2 d_{2} \lambda+\epsilon\left(\lambda^{2}\right) .
$$

Thus, as $\lambda$ goes through zero, a singular point crosses the interface, at the same time changing its type from saddle $(\operatorname{det} J<0)$ to center $(\operatorname{det} J>0$ ). Figure 3 shows the bifurcation diagram for $\nu>0$.

\section{Unfolding the double degeneracy $\lambda=0, \nu=0$}

When both $\lambda$ and $\nu$ are small, the solutions of (35),

$$
\eta=\frac{24 d_{2}}{D}\left(\nu \pm \sqrt{\nu^{2}+\frac{D}{12 d_{2}} \lambda}\right)
$$

are close to the origin. 'l'his represents two singular points that merge and disappear at a bifurcation point satisfying $y^{2}+\left(D / 12 d_{2}\right) \lambda=0$. Close to the bifurcation the two singular points are a saddle and a center. At the bifurcation, they have merged to a cusp point. When $\nu \neq 0$, the results of the previous section hold. Thus, as $\lambda$ crosses zero, one of the singular points (36) crosses the interface and changes its type.

This gives a complete description of the unfolding in the case $D<0$. The resulting bifurcation diagram is shown in Fig. 4.

For $D>0$, similar local bifurcations arise, as shown in Fig. 5. However, the local bifurcations cannot give a com-

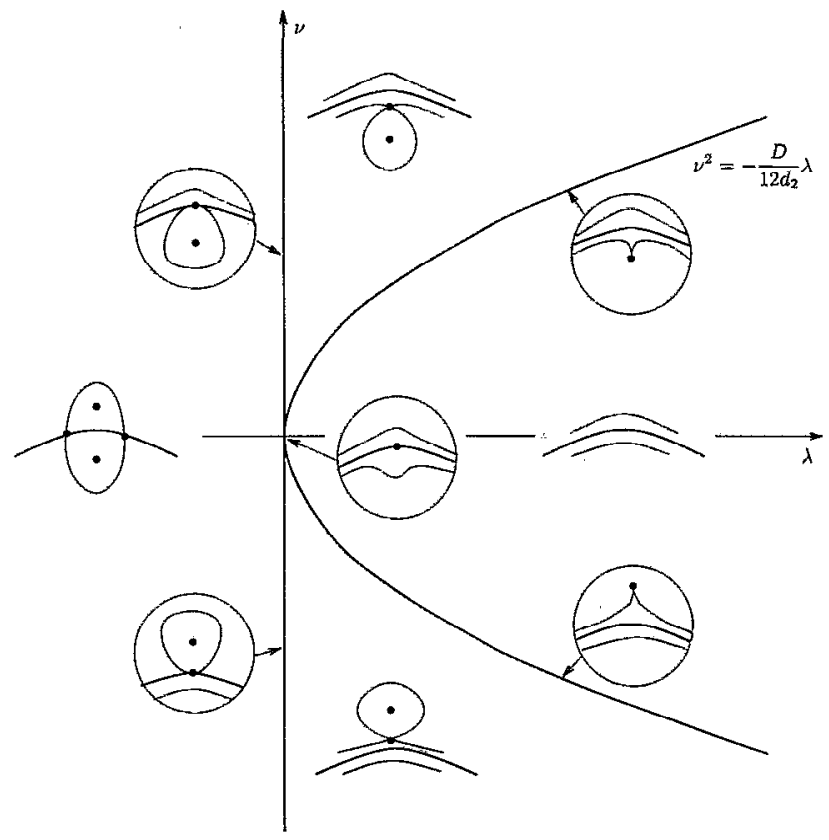

FIG. 4. Unfolding of the double degeneracy $\lambda=\nu=0, D<0$.

plete picture. It appears that the separatrices of the offinterface singular points form a homoclinic loop right after the birth of the points. When $\lambda$ approaches zero from below, there is no homoclinic loop but a heteroclinic cycle connecting the singular points on the interface. Thus, there must be an additional global bifurcation in between.

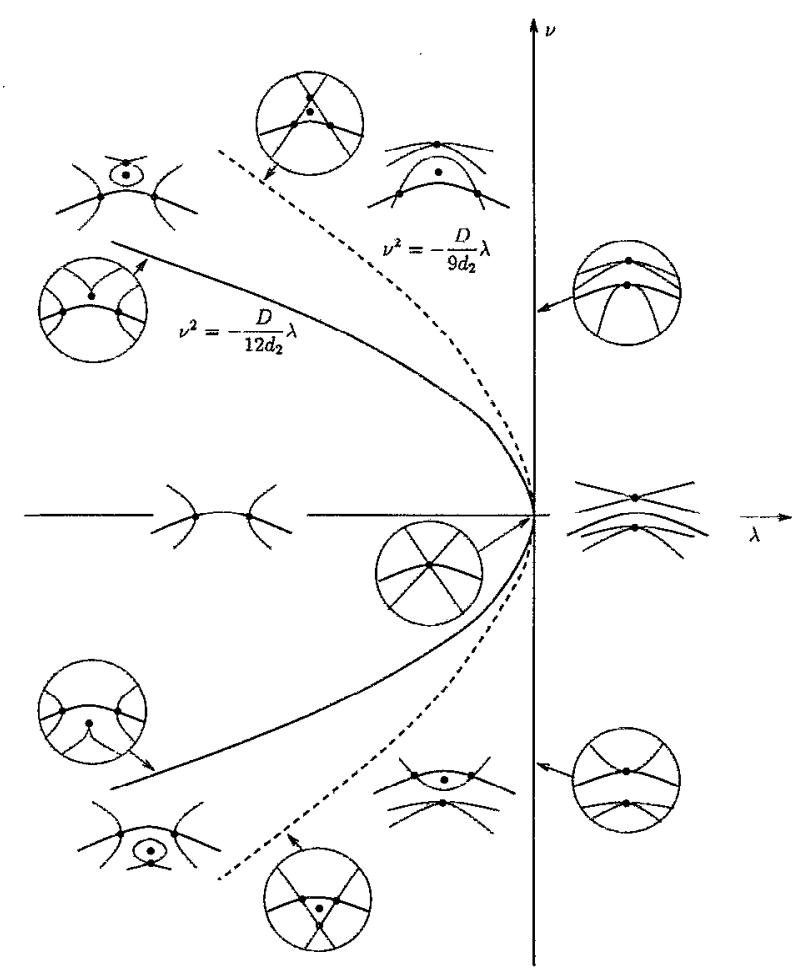

FIG. 5. Unfolding of the double degeneracy $\lambda=\nu=0, D>0$. The dashed line is the global bifurcation curve. 
To find this bifurcation, we consider the streamfunction of the system (27)-(28),

$$
\psi=\eta\left(\lambda+\frac{1}{2} \nu \eta+d_{2} \xi^{2}+\frac{1}{2} a_{11} \xi \eta+\frac{1}{3} a_{02} \eta^{2}\right)
$$

where the level $\psi=0$ contains the interface. Inserting the off-interface saddle point given by (34)-(35) one finds that $\psi=0$ here when

$$
\nu^{2}=-\frac{1}{9} \frac{D}{d_{2}} \lambda
$$

For this combination of parameters, three saddle points are connected in a heteroclinic cycle, and with the bifurcation set given by (38) the bifurcation diagram is complete.

The final case, $D=0$, will not be treated here, since its unfolding depends on third-order terms.

\section{FLOW TOPOLOGY OF THE VISCOUS INTERFACE}

\section{A. The regular case}

To patch the flow field together across the interface, the pressure coefficients $c_{n m}$ in each fluid are first determined from the Navier-Stokes equations (2). Then the dynamic boundary conditions (9)-(11) are used to express the coefficients in fluid 2 in terms of those in fluid 1 . The result is

$$
\begin{aligned}
a_{00}^{(2)}= & a_{00}^{(1)} \\
a_{10}^{(2)}= & a_{10}^{(1)} \\
a_{01}^{(2)}= & \frac{\mu_{1}}{\mu_{2}} a_{01}^{(1)}+2 s_{2} \mu_{R} a_{00}^{(1)}+\frac{\sigma_{1}}{\mu_{2}} \\
a_{20}^{(2)}= & a_{20}^{(1)}-s_{2} \mu_{R}\left(a_{01}^{(1)}+2 a_{00}^{(1)} s_{2}\right)-\frac{s_{2} \sigma_{1}}{\mu_{2}} \\
a_{11}^{(2)}= & \frac{\mu_{1}}{\mu_{2}} a_{11}^{(1)}+2 \mu_{R}\left(3 a_{00}^{(1)} s_{3}-a_{10}^{(1)} s_{2}\right)+\frac{2 \sigma_{2}}{\mu_{2}} \\
a_{02}^{(2)}= & \frac{\mu_{1}}{\mu_{2}} a_{02}^{(1)}+\frac{\rho_{2}-\rho_{1}}{2 \mu_{2}} a_{00}^{(1)} a_{10}^{(1)}+3 \mu_{R}\left(2 a_{00}^{(1)} s_{2}^{2}+a_{01}^{(1)} s_{2}\right. \\
& \left.+a_{20}^{(1)}\right)+\frac{3 s_{3} \sigma_{0}}{\mu_{2}}+\frac{6 s_{2} \sigma_{1}}{\mu_{2}}
\end{aligned}
$$

where

$$
\mu_{R}=\frac{\mu_{1}}{\mu_{2}}-1,
$$

and the surface tension which may vary along the interface has been expanded in a series

$$
\sigma=\sum_{n=0}^{\infty} \sigma_{n} x_{1}^{n} .
$$

Note that the nonlinearity of the Navier-Stokes equations is apparent in Eq. (44).

It follows that if the origin is a regular singular point in fluid $1\left(a_{00}^{(1)}=0, a_{10}^{(1)} \neq 0\right)$, the same holds in fiuid 2 . From (23) we find

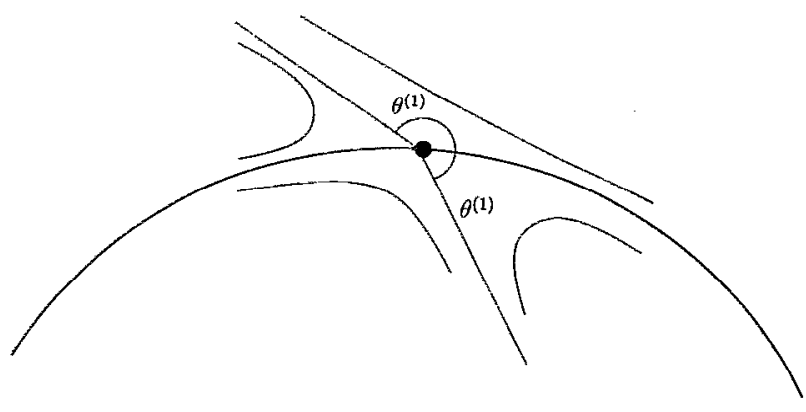

FIG. 6. "Refraction" of a dividing streamline at a regular singular point on the viscous interface.

$$
S=\frac{\tan \theta^{(1)}}{\tan \theta^{(2)}}-\frac{a_{10}^{(1)}}{a_{10}^{(2)}} \frac{a_{01}^{(1)}}{a_{01}^{(2)}}=\frac{\mu_{1}}{\mu_{2}}\left(1+\frac{\sigma_{1}}{\tau_{12}^{(1)}}\right) .
$$

In the absence of a surface tension gradient, (47) is a "refraction law," first discovered by Lugt, ${ }^{8}$ which says that the ratio $S$ of the slopes of the dividing streamlines depends only on the physical properties of the fluids. Surface tension gradients will give a correction that depends on the actual stresses in the interface (Fig. 6).

\section{B. The simple degeneracy}

For the transformed parameters we find

$$
\begin{aligned}
& \lambda^{(2)}=\lambda^{(1)}, \\
& d_{2}^{(2)}=d_{2}^{(1)} .
\end{aligned}
$$

Thus, when the flow is degenerate in one fluid $(\lambda=0)$ the same is the case in the other. Also, the second-order nondegeneracy condition $d_{2} \neq 0$ is valid simultaneously in both fluids.

Here we consider a simple degeneracy in fluid 1 , $\lambda^{(1)}=0, \nu^{(1)} \neq 0$. We find

$$
\nu^{(2)}=\frac{\mu_{1}}{\mu_{2}} \nu^{(1)}+\frac{\sigma_{1}}{\mu_{2}} .
$$

The phase portraits of Fig. 2 may be patched together across the interface as shown in Fig. 7. In the absence of a surface tension gradient $\nu^{(1)}$ and $\nu^{(2)}$ have the same sign, and only cases (a) or (g) are possible, that is, only one fluid can have a dividing streamline. The same will hold for sufficiently small surface tension gradients. The other cases (e) and (f) are possible in the presence of a sufficiently strong surface pressure gradient.

The unfolding of case $(\mathrm{g})$ where the flow in fluid 2 has a dividing streamline, is shown in Fig. 8 . The bifurcation is shown in a global setting where the separatrix in fluid 2 at the degeneracy reconnects to the interface at some stagnation point $S$. Inside the loop formed by the separatrix and the interface at least one singular point must exist. The simplest case is when this is a center, and the loop forms one large eddy.

This picture matches the mean streamlines conjectured by Banner and Melville (Ref. 11, Fig. 2) for the flow over a broken wave. When seen from the rest system of the wave, breaking is signaled by the creation of a stagnation point on 


\section{$\nu^{(1)}>0$}

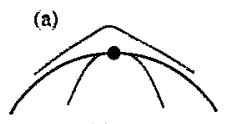

$\nu^{(2)}>0$ (b)

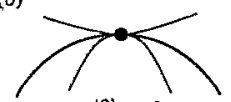

$v^{(2)}<0$
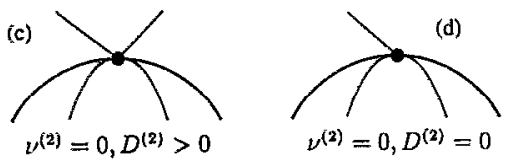

$\nu^{(2)}=0, D^{(2)}=0$

$\nu^{(1)}<0$
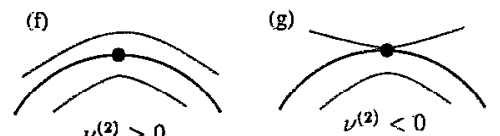

$\nu^{(2)}<0$ (h)

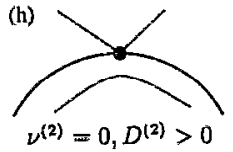

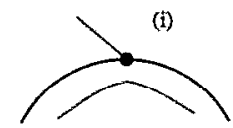

$\nu^{(2)}=0, D^{(2)}=0$

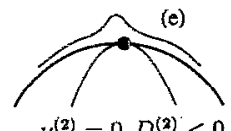

$\nu^{(2)}=0, D^{(2)}<0$
FIG. 7. Flow topologics near the viscous interface when fluid 1 has a simple $\operatorname{degeneracy}\left(\lambda^{(1)}=0, \nu^{(1)} \neq 0\right)$.

the interface. Our bifurcation analysis shows that the simplest possible way this can happen is as shown in Fig. 8. It must, of course, be realized that the breaking of waves is an unsteady process. However, the present study shows that the
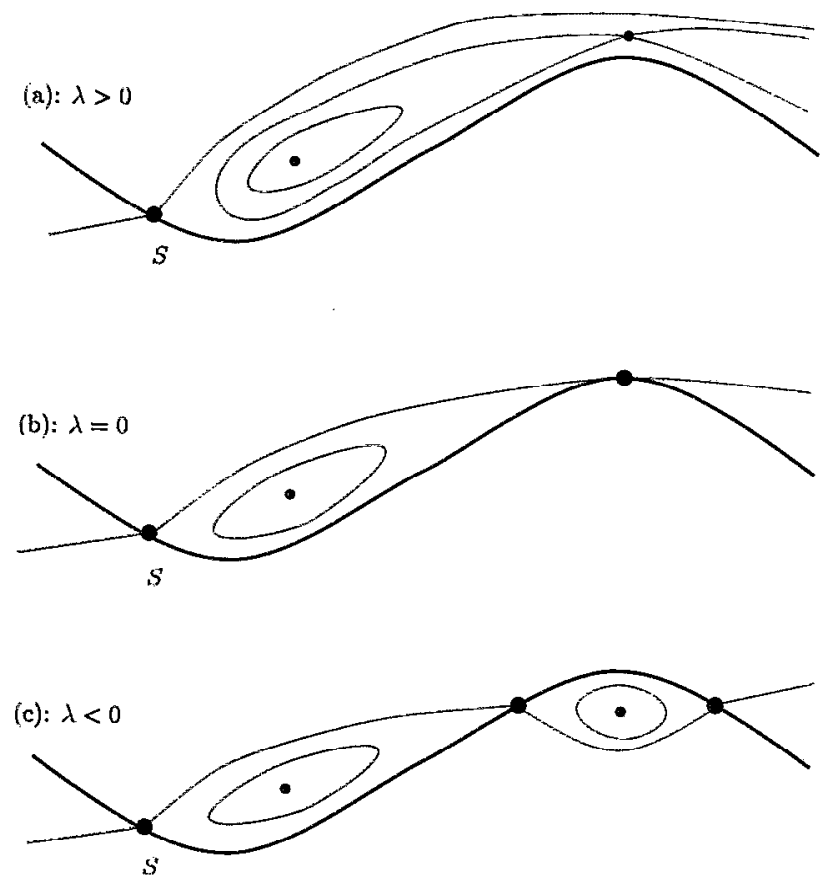

FIG. 8. Unfolding of a degenerate singular point in the case where only fluid 2 separates, case $(\mathrm{g})$ in Fig. 7, here shown in a global context. The heavy line is the interface. (a) Wave prior to breaking. (b) Degenerate configuration. (c) Wave after breaking. toplogy is mainly determined by the equation of continuity. Even if the coefficients $a_{n m}$ are functions of time, the equations for the streamlines must at each instant be of the form (20)-(21). Also, the predictions from a topological investigation are robust in the sense that they do not depend on specific parameter values, but only involve certain inequalities. Thus, one may expect that the mean streamlines agree with the ones found by analyzing a steady flow, which is indeed the case here.

\section{The double degeneracy}

Even if the flow in fluid 1 has only a simple degeneracy, Eq. (50) shows that the flow in fluid 2 can have a double degeneracy. Returning to physical variables, we find that $\nu^{(2)}=0$ when

$$
\sigma_{1}=-\tau_{12}^{(1)},
$$

that is, when tangential stress in fluid 1 balances the surface tension gradient. The flow in fluid 2 then depends on the sign of the parametcr $D$ defined in (33). We find

$$
\begin{aligned}
D^{(2)}= & 9 a_{11}^{(2) 2}-48 a_{02}^{(2)} d_{2}^{(2)} \\
= & \left(3 \frac{\mu_{1}}{\mu_{2}} a_{11}^{(1)}+6 \frac{\sigma_{2}}{\mu_{2}}\right)^{2}-48 d_{2}^{(1)}\left(\frac{\mu_{1}}{\mu_{2}} a_{02}^{(1)}+3 \mu_{R} d_{2}^{(1)}\right. \\
& \left.+3 \frac{s_{3} \sigma_{0}}{\mu_{2}}+6 \frac{s_{2} \sigma_{1}}{\mu_{2}}\right) .
\end{aligned}
$$

From the available information, nothing can be said about the sign of $D^{(2)}$, and consequently all of the configurations (c) -(e) and (h)-(j) in Fig. 7 may occur. We can also find the unfoldings of these degenerate configurations-except for the cases with $D=0$-by patching together the bifurcation diagrams in Figs. 3-5. This is a straightforward procedure, and as an example we consider the unfolding of case (e). Here the natural parameters to vary are $\lambda^{(1)}=\lambda^{(2)}=\lambda$ close to zero, and the surface tension gradient close to its degenerate value, $\sigma_{1}=\mu_{1} \nu_{1}$. The result is shown in Fig. 9 .

The flows in both fluids have a double degeneracy if $\sigma_{1}=0$. In that case, any of the 9 possible combinations of the degenerate flows in Fig. 2 on each side of the interface may occur, since any combination of signs of $D^{(1)}$ and $D^{(2)}$ is possible. Again, for the four cases with $D^{(1)}, D^{(2)}$ $\neq 0$, the unfoldings can be found from combinations of the Figs. 4 and 5 .

\section{FLOW TOPOLOGY OF THE FREE SURFACE}

\section{A. Regular and degenerate singular points}

Below a free surface, the boundary conditions (13)-(14) give the following relations between the coefficients:

$$
\begin{aligned}
& a_{01}=-\left(2 a_{00} s_{2}+\frac{\sigma_{1}}{\mu}\right), \\
& a_{11}=2 a_{10} s_{2}-6 a_{00} s_{3}-2 \frac{\sigma_{2}}{\mu}, \\
& a_{02}=\frac{\rho}{2 \mu} a_{00} a_{10}-3 a_{20}-\frac{3}{\mu}\left(s_{2} \sigma_{1}+s_{3} \sigma_{0}\right) .
\end{aligned}
$$




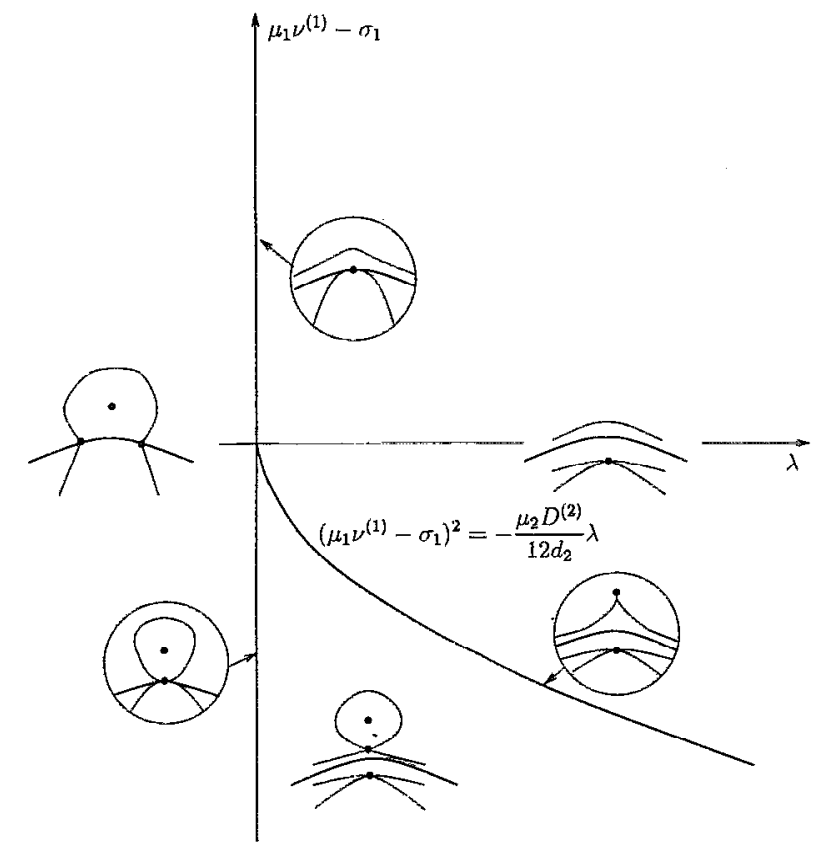

FIG. 9. Unfolding of the degeneracy (e) from Fig. 7.

If $a_{00}=0$, the origin is a singular point. If $a_{10} \neq 0$, the point is nondegenerate, and a dividing streamline as in Fig. 2(a) exists. The slope of the streamline is found from Eq. (23),

$$
\tan \theta=2 \frac{\mu}{\sigma_{1}} a_{10}=\frac{\tau_{11}}{\sigma_{1}},
$$

and $\theta=\pi / 2$ if $\sigma_{1}=0$. In particular, if the surface tension is constant, the dividing streamline is perpendicular to the interface.

In the degenerate case, $a_{00}=a_{10}=0$, we find

$$
a_{01}=-\frac{\sigma_{1}}{\mu},
$$

and we get a simple degeneracy, $a_{01} \neq 0$, only if $\sigma_{1} \neq 0$. In that case, the toplogy of the flow is as shown one of the sides of the interface in Fig. 2(b). If $\nu$ and $d_{2}$ have the same sign, the topology is as below the surface in the figure, while the other side is relevant if the parameters have opposite signs.

If in addition $\sigma_{1}=0$, the origin is a double degenerate singular point. The topology is determined by the sign of

$D=9 a_{11}^{2}-48 a_{02} d_{2}=36\left(\frac{\sigma_{2}}{\mu}\right)^{2}+144 a_{20}\left(a_{20}+\frac{s_{3} \sigma_{0}}{\mu}\right)$.

Since $D$ may bc of any sign, all configurations in Figs. 2(c)-2(e) may occur. This generalizes a result by $\operatorname{Lugt}^{8}$ who considered a circular surface. Here $s_{3}=0$, and only $D>0$ is possible.

The vorticity at the origin is easily computed as

$$
\omega=4 s_{2} a_{00}+\frac{\sigma_{1}}{\mu},
$$

which generalizes "surface vorticity equals twice the curvature times tangential surface velocity" (Ref. 14, p. 366), to include surface tension gradients. Moreover, for the vorticity flux we get

$$
\mu(\nabla \omega) \cdot \boldsymbol{n}=-\rho a_{00} a_{10}+4 \mu a_{20}+6\left(s_{3} \sigma_{0}+s_{2} \sigma_{1}\right)
$$

which generalizes a result by Lugt [Ref. 8, Eq. (39)] to cover surface tension gradients and change of curvature of the surface.

The unfoldings of the degeneracies are immediately found from the unfoldings described in Sec. III.

\section{B. Flow below a stagnant film}

In the steady flow below a stagnant film of surfaceactive material, variation of the surface tension along the film may give rise to a small disturbance of the surface-the Reynolds ridge. The asymptotic theory of Harper and Dixon ${ }^{15}$ gives a good description of the actual shape of the surface, ${ }^{12}$ but gives no detailed information on the flow topology. Here we consider the problem from the topological point of view.

Assuming the distribution of surface matter to be smooth and to give rise to a smooth distribution of surface tension, the theory of the previous section applies. If vertical diffusion of surface matter is neglected, the surface must be stationary in a steady flow. This imposes the further boundary condition at the surface,

$$
t_{i} u_{i}=0 \text {. }
$$

Since the surface for (24)-(25) is given by $\eta=0$, we immediately get, taking $x_{0}=0$,

$$
d_{2}=a_{10}=a_{00}=0 .
$$

Furthermore, from the boundary conditions (52)-(54) and the definition (26) of $d_{2}$ we get

$$
a_{01}=\cdots \frac{\sigma_{1}}{\mu}, \quad a_{11}=\frac{2 \sigma_{2}}{\mu}, \quad a_{02}=-3 \frac{2 s_{2} \sigma_{1}+s_{3} \sigma_{0}}{\mu} .
$$

With this, the equations for both $\dot{\xi}$ and $\dot{\eta}$ will have a factor $\eta / \mu$ on the right-hand side. Since division by a common factor does not change the trajectories, we get the equations for the streamlines

$$
\begin{aligned}
& \dot{\xi}=-\sigma_{1}-2 \sigma_{2} \xi-3\left(2 s_{2} \sigma_{1}+s_{3} \sigma_{0}\right) \eta, \\
& \dot{\eta}=\sigma_{2} \eta .
\end{aligned}
$$

We see that the origin is a stagnation point only if $\sigma_{1}=0$. The eigenvalues are $-2 \sigma_{2}$ and $\sigma_{2}$ with eigenvectors $(1,0)$ and $\left(s_{3} \sigma_{0},-\sigma_{2}\right)$ respectively, when $\sigma_{2} \neq 0$. Thus, when $\sigma_{2}>0$ the dividing streamline in the fluid is the unstable separatrix of the stagnation point, and the flow diverges from the surface here. If $\sigma_{2}<0$, the dividing streamline is the stable separatrix, and the flow attaches to the surface. Phrased differently: The flow below a stagnant film separates where the surface tension has a local minimum and attaches where the surface tension has a local maximum.

We also find the slope of the dividing streamline as 


$$
\tan \theta=-\frac{\sigma_{2}}{s_{3} \sigma_{0}} .
$$

\section{ACKNOWLEDGMENTS}

This work was initiated while the author visited University of California at San Diego in the summer of 1992. The subject was suggested by Hassan Aref, and I am indebted to his constant encouragement and inspiration. Financial support from Office of Naval Research under Grant No. N00014-92-J-1610 and The Danish Natural Science Research Council is gratefully acknowledged.

'H. K. Moffatt and A. 'Isinober (editors), Topological Fluid Dynamics (Cambridge University Press, Cambridge, 1989).

${ }^{2} \mathrm{~K}$. Oswatitsch, "Die Ablösungsbedingung von grenzschichten," in IUTAM Symposium on Boundary Layer Research, edited by H. Görtler (Springer, Berlin, 1958), p. 357.

"R. Legendre, "Séparation de l'écoulement laminaire tridimensionel," Res. Aéro. 54, 3 (1956).

${ }^{4}$ M. J. Lighthill, in Laminar Boundary Layers, edited by L. Rosenhead (Oxford University Press, London, 1963), p. 72.
${ }^{5} \mathrm{M}$. Tobak and D. J. Peake, "Topology of three-dimensional separated Hows," Annu. Rev. Fluid Mech. 14, 207 (1986).

${ }^{6}$ P. G. Bakker, Bifurcations in Flow Patterns (Klüwer, Dordrecht, 1991).

${ }^{7}$ A. E. Perry and M. S. Chong, "A series-expansion study of the NavierStokes equations with application to three-dimensional separation patterns," J. Fluid Mech. 173, 207 (1986).

${ }^{8}$ H. J. Lugt, "Local properties at a viscous free surface," Phys. Fluids 30, 3647 (1987).

${ }^{9} \mathrm{H}$. J. Lugt, "Oblique vortices on a solid wall and on an interface between two immiscible viscous fluids," Phys. Fluids A 1, 1424 (1989).

${ }^{10} \mathrm{U}$. Dallmann, "Three-dimensional vortex structures and vorticity topology," Fluid Dyn. Res. 3, 183 (1988).

${ }^{11}$ M. L. Banner and W. K. Melville, "On the separation of air flow over water waves," J. Fluid Mech. 77, 825 (1976).

${ }^{12} \mathrm{~J}$. C. Scott, "Flow beneath a stagnate film on water: The Reynolds ridge," J. Fluid Mech. 116, 283 (1.982).

${ }^{13}$ A. A. Andronov, E. A. Leontovich, 1. 1. Gordon, and A. G. Maier, Qualitative Theory of Second-Order Dynamical Systems (Wiley, New York, 1973).

${ }^{14} \mathrm{G}$. K. Batchelor, An Introduction to Fluid Dynamics (Cambridge University Press, Cambridge, 1967).

${ }^{15}$ J. F. Harper and J. N. Dixon, "The leading edge of a surface film on contaminated flowing water," in Proceedings of the 5th Australasian Conference on Hydraulics and Fluid Mechanics, Christchurch, New Zealand, 1974 , p. 499. 